\title{
Sôbre as ordens infinitesimais
}

\author{
Frederico Pimentel Gomes
}

Assistente interino da 16.9 cadeira

\section{INDICE}

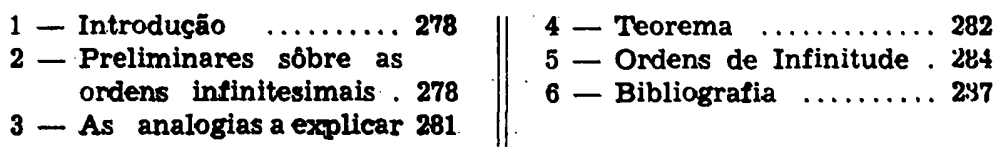


1. - INTRODUÇAO - Muitos dos que estudaram os infinitésimos, também chamados infinitamente pequenos, devem ter notado a curiosa analogia entre os teoremas sobre as ordens infinitesimais dos produtos, quocientes, potencias e raizes de infinitésimos e as propriedades dos logaritmos.

O autor dêste trabalho, preocupado com essa interessante analogia, chegou a demonstrar um teorema que relaciona as ordens infinitesimais aos logaritmos, deixando o assunto perfeitamente esclarecido.

2. - PRELIMINARES SOBRE AS ORDENS INFINITESIMAIS - Sejam $\mathbf{y}, \mathbf{z}, \mathbf{u}, \mathbf{t}$, etc. funçסes reais de uma variável real $\mathbf{x}$. Se tivermos :

$\lim _{x \rightarrow r} y=0, \lim _{x \rightarrow r} z=0, \lim _{x \rightarrow r} u=0, \quad \lim _{x \rightarrow r} t=0$,

e assim por diante, entăo $\mathbf{y}, \mathbf{z}, \mathbf{u}, \mathbf{t}$, etc. são, por definição, funções infinitésimas ou infinitésimos ou infinitamente pequenos no ponto $r$.

Dal se conclui que o conceito de infinitésimo está estreitamente ligado aos de funçăo e limite.

Suponhamos que tomamos o infinitésimo y para termo de comparação; y é entăo o nosso infinitésimo principal.

$(2,1)$ Ainda por definiçăo, se tivermos:

$$
\lim _{x \rightarrow r} \frac{|z|}{|y|^{P}}=k
$$

sendo $k$ um número finito e diferente de zero, diremos que $z$ é de ordem p em rẻlaçáo ao infinitésimo principal y. Isto se pode indicar pela seguinte notaçăo:

$$
\operatorname{ord}_{\mathbf{y}} \mathrm{z}=\mathrm{p},
$$

sendo $\mathbf{p}$ um número real positivo.

De (1) se conclui que

$$
\frac{|z|}{|y|^{p}}=k+t
$$

sendo t um infinitésimo no ponto $r$. 
E fácil demonstrar que, sendo z de ordem $p$ em relaçáo a J e sendo $p>q$, temos:

$$
\lim _{x \rightarrow r} \frac{|x|}{|y|^{9}}=0
$$

o que indica que $z$ é de ordem superior a $q$.

Se, pelo contrário, $p<q$, temos :

$$
\lim _{x \rightarrow r} \frac{|z|}{|y|^{9}}=+\infty
$$

o que indica que $z$ é de ordem inferior a q.

As vezes a relaçáo $\frac{|z|}{|y| p}$ náo tende para nenhum limite quando $x$ tende para $r$, embora se mantenha constantemente entre dois valores finitos e do mesmo sinal. o que acontece, por exemplo, se tomarmos

$$
z=\left(3+\operatorname{sen} \frac{1}{x}\right) x^{2}
$$

e $y=x$, infinitésimos no ponto zero.

Fica :

$$
\frac{|z|}{|y|^{2}}=3+\operatorname{sen} \frac{1}{x}
$$

O segundo membro nåo tende para nenhum limite quando $x$ tende para zero. Mas se mantém constantemente finito e năo nulo, pois podemos escrever :

$$
2 \leq \frac{|z|}{|y \cdot|^{2}} \leq 4
$$

2,2) NIEWENGLOWSKI estende a esse caso, acompanhando CAUCHY, o conceito de ordem infinitesimal como se segue. Sendo $h>0$, podemos escrever :

$\lim _{x \rightarrow r} \frac{|z|}{|y|^{2}+12}=+\infty$

$$
\lim _{x \rightarrow r} \frac{|x|}{|y|^{2-1}}=0
$$


Portanto a ordem infinitesimal p do infinitésimo a em relaçáo a y é tal que

$$
2 \div \mathrm{h}<\mathrm{p}<2+\mathrm{h} .
$$

Como $\mathbf{h}$ pode ser táo pequeno quanto se queira, pode-se to$\operatorname{mar} p=2$ como limite.

Poderiamos, sem muita dificuldade, estender a ésse caso a definição de ordem infinitesimal sem ser preciso recorrer a uma nova passagem ao limite. Basta, para isso, definir como se segue a ordem infinitesimal.

$(2,3)$ Sendo $\mathrm{z}$ e $\mathrm{y}$ funçzes infinitésimas de $\mathrm{x}$ no ponto $\mathrm{r}$, diz-se que z é de ordem $\mathbf{p}$ em relaçáo a y quando podemos escrever :

$$
\frac{|\mathrm{z}|}{|\mathrm{y}| \mathrm{p}}=\mathrm{F}(\mathrm{x})+\mathrm{t}
$$

sendo $\mathbf{t}$ um infinitésimo no ponto $\mathbf{r}$ e $F(x)$ tal que permita que se escreva :

$$
\mathbf{m}<\mathrm{F}(\mathrm{x})<\mathbf{n}
$$

nas vizinhanças do ponto $r$, sendo $m$ e $n$ números finitos e positivos, e sendo ainda considerada apenas a determinaçăo real positiva de $|\mathrm{y}| \mathrm{p}$.

Como caso particular podemos ter :

$$
\mathbf{F}(\mathbf{x})=\mathbf{k}
$$

sendo k uma constante.

No caso das funçøes complexas de uma varlável complexa ou funções vectoriais de um escalar ou vector, o conceito de ordem infinitesimal se reduz ao caso anterior pela consideraçáo de seus módulos, e portanto nossa última definiçáo continua de pé.

Por exemplo a variável complexa $\mathrm{z}=\mathrm{x}+\mathrm{yl}, \operatorname{com} \mathrm{x}$ e $\mathrm{y}$ reais, é infinitésima no ponto $(0,0)$, pols temos

$$
|z|=\sqrt{x^{2}+y^{2}}
$$

$$
\begin{array}{ll}
\lim _{\substack{x \rightarrow \sigma^{\prime} \\
y \rightarrow 0}}|z|=0 \quad \text { e esereve-se } & \lim _{\substack{x \rightarrow 0 \\
y \rightarrow 0}} z=0 \text { oul } z \rightarrow 0 \\
&
\end{array}
$$


t evidente que, sendo $F(z)=f(z)+1 . g(z)$ se $F(z)$ for infinitésima em $z_{0}$, entăo $f(z)$ e $g(z)$ seráo infinitésimas em $z_{v}$, e reciprocamente.

Por exemplo a funçåo complexa $y=F(z)=z^{2}+z^{3} e ́ d e$ segunda ordem em relação a $z$, pois temos :

$$
\begin{gathered}
\frac{\left|z^{2}\right|-\left|z^{3}\right|}{|z|^{2}} \leq \frac{\left|z^{2}+z^{3}\right|}{|z|^{2}} \leq \frac{\left|z^{2}\right|+\left|z^{3}\right|}{|z|^{2}} \\
1-|z| \leq \frac{\left|z^{2}+z^{3}\right|}{|z|^{2}} \leq 1+|z|
\end{gathered}
$$

$\therefore \lim _{z \rightarrow 0} \frac{\left|z^{2}+z^{3}\right|}{|z|}=1$ ou $\frac{\left|z^{2}+z^{3}\right|}{|z|^{2}}=F(x)+t$, sendo $x=|z|$ e $1 / 2<F(x)<3 / 2$ para $x<1 / 2$.

Sendo x uma variável real, a funçăo vectorial

$v=x^{3} i+x i+\sqrt{x} k$ é de ordem $1 / 2$ em relaçăo a $x$, pois temos

$$
\begin{gathered}
\frac{|x|^{1 / 2}-\left|x^{3}\right|-|x|}{|x|^{1 / 2}} \leq \frac{|v|}{|x|^{1 /:}} \leq \frac{|x|^{3}+|x|+|x| 1 / 2}{|x|^{1 / 2}} \\
1-\frac{|x|^{3}+|x|}{|x|^{1 / 2}} \leq \frac{|v|}{|x|^{1 / 2}} \leq 1+\frac{|x|^{3}+|x|}{|x|^{1 / 2}} \\
\therefore \lim _{x \rightarrow 0} \frac{|v|}{|x|^{1 / 2}}=1 \text { ou } \frac{|v|}{|x|^{1 / 2}}=F \text { (x) tt, } \\
\text { sendo } \frac{1}{5}<F(x)<\frac{7}{4} \text { para }|x|<\frac{1}{4} .
\end{gathered}
$$

3. - As ANALOGIAS A EXPLICAR - szo as que se seguem, nas quais além da anotaçáo já exposta para a ordem ininfinitesimal, utilizaremos a notaçáo comum $\left(\log _{a}\right.$ b) para 0 logaritmo real de b na base a $>0$. 
a ) Produto de infinitésimas z, $\mathbf{u}, \mathbf{t}$, etc.

$\operatorname{ord}_{v}(z . u . t \ldots)=\operatorname{ord}_{y} u+\operatorname{ord}_{y} z+\operatorname{ord}_{y} t+\cdots$

a') Produto de números pasitivos quaisquer $b, c, d$, etc.

$\log _{a}$ (b. c. d. ..) $=\log _{a} b+\log _{a} e+\log _{a} d+\ldots$

b) Quociente de dois infinitésimos z e u.

$$
\operatorname{ord}_{y} \frac{z}{u}=\operatorname{ord}_{y} z-\operatorname{ord}_{y} u
$$

b') Quociente de dois númros positivos quaisquer b e c.

$$
\log _{a} \cdot \frac{b}{c}=\log _{a} b-\log _{a} c
$$

c ) Poténcia de expoente $m$ de um infinitésimo a.

$$
\operatorname{ord}_{y} z^{m}=m \operatorname{ord}_{y} z
$$

c') Potencia de expoente $\mathbf{m}$ de um número positivo qualquer $b$,

$$
\log _{\mathbf{a}} \mathbf{b}^{m}=\mathbf{m} \log _{\mathbf{a}} \dot{b}^{\dot{2}} \text {. }
$$

d) Raiz de indice $n$ de um infinitésimo $z$.

$$
\operatorname{ord}_{y} \sqrt[n]{z}=\frac{1}{n} \operatorname{ord}_{y} z
$$

d') Raiz de indice $n$ de um numero positivo qualquer $b$.

$$
\log _{a} \sqrt[n]{b}=\frac{1}{n} \log _{a} b
$$

A analogia é perfeita, como se vê. Qual a sua razáo ? O teorema seguinte esclarece bem o motivo.

4. TEOREMA - Se a é um infinitésimo de ordem q em relaçăo a y,z y y sendo funçסes de $x$ infinitésimas no ponto $r$, entăo existe o limite do logaritmo de $|\mathrm{z}| \mathrm{na}$ base $|\mathrm{y}|$ e esse llmite é igual a q.

Tese: Se ordy $z=q$, entz̃o $\lim _{x \rightarrow r} \log |y|=q$ 
Demonstração - Seja, por hipótese, e de acórdo com as convenções estabelecidas :

$$
\frac{|\mathrm{z}|}{|\mathrm{y}|^{\mathbf{q}}}=\mathrm{F}(\mathrm{x})+\mathrm{t}
$$

Vamos demonstrar que existe

$$
\lim _{x \rightarrow r} \log _{|y|}|z|=q
$$

Podemos escrever :

$\log _{a}|z|=q \log _{a}|y|+\log _{a}[F(x)+t]$, com a $\neq 1$ positivo.

$$
\therefore \frac{\log _{a}|\mathrm{z}|}{\log |\mathrm{y}|}=\mathrm{q}+\frac{\log _{\mathrm{a}}[\mathrm{F}(\mathrm{x})+\mathrm{t}]}{\log _{\mathrm{a}}|\mathrm{y}|}
$$

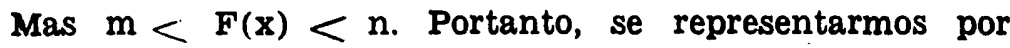
u o valor absoluto do segundo têrmo do segundo membro da última igualdade, poderemos escrever, sendo $\mathrm{A}$ o maior dos logaritmos de m e n, em valor absoluto :

$$
0<u<\left|\frac{A}{\log _{a}|y|}\right|
$$

Mas

$$
\lim _{x \rightarrow r} \frac{A}{\log _{a}|y|}=0
$$

Portanto

$$
\lim _{x \rightarrow r} u_{1}=0
$$

* fácil concluir que

$$
\begin{gathered}
\lim _{x \rightarrow r} \frac{\log _{a}|z|}{\log _{a}|y|}=q \\
\operatorname{Mas} q=\operatorname{ord}_{y} z e_{1} \frac{\log _{a}|z|}{\log _{a}|y|}=\log _{|y|}|z|
\end{gathered}
$$


Portanto :

$$
\operatorname{ord}_{y} \mathbf{z}=\lim _{\mathbf{x}-\mathbf{r}} \log _{|\mathbf{y}|}|\mathbf{z}| \quad \text { C. Q. D. }
$$

A reciproca désse teorema, porém, não é verdadeira, isto é, a existência de lim $\log |z|=p$ não implica necessàriamen-

$$
\mathrm{x} \rightarrow 0 \quad \mid \mathrm{y} i
$$

te que a ordem de $z$ em lelação a y tal como foi definida em $(2,3)$, seja p. Mas é verdadeira se tomarmos por base a definição $(2,2)$.

Por exemplo, seja $z=x^{2} \log x, y=x$.

Temos :

$$
\lim _{x \rightarrow 0} \log _{|y|}|z|=\frac{2 \log |x|+\log \log |x|}{\log |x|}=2
$$

No entanto

$$
\lim _{x \rightarrow 0} \frac{|z|}{|y|^{2}}=\lim _{x \rightarrow 0}|\log x|=+\infty
$$

e portanto $z$ năo é de segundo ordem em relaçăo a $x$ segundo $(2,3)$, mas o é segundo $(2,2)$, conforme o leitor poderá verificar.

Demonstrado êste teorema, os teoremas comuns sôbre produtos, quocientes, poténcias e raizes de infinitésimos podem ser expressos por um corolário dêle.

COROLARIO - As ordens infinitesimais dos produtos, quocientes, potências e raizes de infinitésimos obedecem às mesmas propriedade correspondentes dos logaritmos.

5. - ORDENS DE INFINITUDE - Sendo $Y$ uma função de $x$ tal que

$$
\lim _{\mathbf{X} \rightarrow \mathbf{r}} \mathbf{Y}= \pm \infty
$$
to $\mathbf{r}$.

diz-se que $Y$ é uma função infinita ou um infinito. no ponPode-se definir a ordem de infinitude de um infinito $\mathrm{Z}$ em 
relaçăo a um infinito principal Y. O intinito $Z$ será de ordem $\mathbf{P}$ em relaçăo a $Y$ se pudermos escrever :

$$
\lim _{x \rightarrow r} \frac{|Z|}{|Y| p}=k
$$

sendo $\mathrm{k}$ um numero finito e diferente de zero.

De uma maneira mais geral, pode-se dizer que $z$ e de ordem $p$ em relaçăo a $Y$ se, sendo $h>0$, tivermas :

$$
\lim _{x \rightarrow r} \frac{|Z|}{|Y|^{p-h}}=+\infty \quad \text { e } \lim _{x \rightarrow r} \frac{|Z|}{|Y|^{p \div-h}}=0
$$

De um modo um pouco menas geral poderfamas dizer que $\mathrm{Z}$ é de ordem p em relaçáo a $Y$ no ponto $\mathrm{r}$ se pudermas escreyr :

$$
\frac{|Z|}{|Y| p}=F(x)+t
$$

sendo $t$ um infinitesimo no ponto r e $F(x)$ tal que se possa escrever :

$$
\mathbf{m}<\mathbf{F}(\mathbf{x})<\mathbf{n},
$$

sendo $\mathrm{m}$ e $\mathrm{n}$ numeros finitos e pasitivos.

Como caso particular temos aquele em que $F(x)$ é igual a uma constante $\mathbf{k}$.

Mas essas novas definiçoes săo desnecessárias, pois a ordem de infinitude dos infinitos pode ser redusida facllmente ordem infinitesimal dos infinitésimas. Pols se $Z$ e $\mathbf{Y}$ sto infinitos, $\frac{1}{7}$ e $\frac{1}{Y}$ săo infinitésimas. $E$ a ordem de infinitude de $\mathrm{Z}$ em relaçáo a $Y$ corresponde exatamente $a$ ordem infinitasimal de $\frac{1}{7}$ em $\quad$ relaçzo a $\frac{1}{Y}$

$$
\begin{aligned}
& \text { Seja } \frac{1}{Y}=y \text { e podemos escrever : } \\
& \operatorname{ord}_{y} \frac{1}{Z}=\lim _{x \rightarrow r} \quad \log _{|y|}\left|\frac{1}{Z}\right| \\
& =-\lim _{x \rightarrow r} \quad \log _{|y|}|Z| \\
& =-\operatorname{ord}_{y} Z
\end{aligned}
$$


Anàlogamente, sendo z um infinitésimo temos :

$$
\operatorname{ord}_{y} \frac{1}{z}=-\operatorname{ord}_{y} z \text {. }
$$

Daí se conclui que a ordem de infinitude não é mais do que uma ordem infinitesimal negativa.

Portanto a ordem infinitesimal, considerada de um modo bem geral, pode ter qualquer valor real, positivo ou negativo.

A ordem infinitesimal nula corresponde às funçóes finitas e não nulas.

Sim; pois sendo s uma função de $\mathrm{x}$ não infinitésima e não infinita no ponto $\mathbf{r}$, podemos escrecer :

$$
\frac{|\mathrm{s}|}{\mathrm{y}^{0}}=\mathrm{F}(\mathrm{x})+\mathrm{t}
$$

pois dal tiramos :

$$
|\mathrm{s}|=\mathrm{F}(\mathrm{x})+\mathrm{t} .
$$

e como estão excluidos os casos de

$$
\lim _{x \rightarrow r} s=0 \quad \text { e } \lim _{x \rightarrow r} s= \pm \infty ;,
$$

evidentemente teremas nas vizinhanças de $\mathbf{r}$ :

$$
\mathbf{m}<|\mathrm{s}|<\mathbf{n}
$$

șe $s$ for uma função fịnita e não nula.

$\mathbf{E}$ como $\mathbf{t}$ é infinitésimo no ponto $\mathbf{r}$, podemos escolher um entôrno de $\mathbf{r}$ tal que

$$
|t|<\frac{m}{2} \text { e }|t|<\frac{n}{2} .
$$

de onde se conclui que

$$
\frac{\mathrm{m}}{2}<\mathrm{F}(\mathrm{x})<\mathrm{n}
$$




$$
\begin{aligned}
& \text { no caso de } t>0 \\
& m<F(x)<\frac{3 n}{2} \\
& \text { no caso de } t<0
\end{aligned}
$$

Em ambos os casos s será de ordem zero em relaçăo ao infinitésimo y, como queriamos demonstrar.

\section{6 - BIBLIOGRAFIA}

a) CARNEIRO, Orlando - Apontamento de aula.

b) COMBEROUSSE, Charles de - Cours de Mathématiques. 1929.

c) FRANKIIN, Philip - A Treatise on Advanced Calculus. 1940.

d) NIEWENGLOWSKI, B. - Cours d'Algèbre. 1931.

e) TOSELLO, Andre - Apostlias.

f) WOODS, Frederick S. - Advanced Calculus. S/ data.

g) BOREL, tmile - Leçons sur la Théorie de la Croissance. 1910.

h) SEVERI, Francesco - Lezionl di Analisi. 1938. 